\title{
Glicemia do sangue capilar e venoso de cães saudáveis: mensuração por método eletroquímico versus enzimático laboratorial
}

\section{Capillary and venous blood glucose of healthy dogs: measurement by electrochemical method versus enzymatic laboratory}

\author{
Patricia Almeida Ferreira ${ }^{1 *}$; Eduardo Negri Mueller²; Elisângela Coelho Fischer³; \\ Mariana Teixeira Tillmann ${ }^{1}$; William Peres ${ }^{4}$; Márcia de Oliveira Nobre ${ }^{5}$
}

\section{Resumo}

\begin{abstract}
A avaliação da glicose com o uso de glicosímetro é importante pela rapidez e custo, sendo um importante instrumento para o controle de alterações da glicemia e permitindo que decisões diagnósticas e terapêuticas sejam tomadas rapidamente. Neste trabalho objetivou-se aferir e comparar a glicemia no sangue capilar e no sangue venoso de cães hígidos com uso de glicosímetro digital portátil, tendo como padrão de referência os valores obtidos pelo método enzimático laboratorial com amostras de sangue venoso. Foi avaliada a glicemia de 20 cães sem raça definida, mantidos em jejum alimentar de 12 horas. Para avaliação da glicemia com glicosímetro digital portátil foi utilizada uma gota de sangue capilar da margem da orelha e da veia cefálica, enquanto que para dosagem da glicemia pelo método enzimático laboratorial foram colhidos dois $\mathrm{mL}$ de amostra venosa. Os valores médios da glicose sanguínea aferida por meio do glicosímetro digital portátil em amostras de sangue periférico capilar e venoso foram, respectivamente, $84,60 \mathrm{mg} / \mathrm{dL}( \pm 11,15)$ e $76,65 \mathrm{mg} / \mathrm{dL}( \pm 10,73)$, enquanto que pelo método enzimático laboratorial foi $80,7 \mathrm{mg} / \mathrm{dL}( \pm 9,97)$, não sendo demonstradas diferenças estatísticas significativas. Em relação ao método laboratorial, a variação da glicemia obtida pelo glicosímetro no sangue capilar foi de $9,4 \%$ e no venoso de $10,4 \%$. Conclui-se que o glicosímetro pode ser utilizado com segurança em cães, já que as médias dos valores da glicemia capilar e venosa, aferidas por glicosímetro digital portátil, foram similares aos valores obtidos pelo método laboratorial.
\end{abstract}

Palavras-chave: Glicose, glicosímetro, espectrofotometria

\begin{abstract}
Glucose evaluation with glucometer is important due to its high speed and low cost, being an important instrument to control glycemia alterations and allowing quick diagnostic and therapeutic decisions. In this work we have measured and compared the glycemia of capillary and venous blood of healthy dogs through digital glucometer, using, as reference, the values obtained by laboratorial enzymatic method with samples of venous blood. It was evaluated the glycemia of twenty dogs, of no specific breed, fasting for 12 hours. To evaluate the glycemia, with digital glucometer, it was used one blood drop from the ear's border and cephalic vein, while for the evaluation through laboratorial enzymatic method it was collected $2 \mathrm{~mL}$ of blood from the cephalic vein. The glycemia average values, obtained by digital glucometer, from venous and capillary blood were, respectively, 84,60 mg/dL $( \pm 11,15)$ and $76,65 \mathrm{mg} /$
\end{abstract}

\footnotetext{
${ }^{1}$ Discente(s) de Doutorado, Programa de Pós-Graduação Veterinária, Universidade Federal de Pelotas, UFPel, Pelotas, RS. E-mail: pitiferreira@gmail.com; marianatillmann@yahoo.com.br

2 Prof. Dr. do Instituto Federal Catarinense, Campus Concórdia, Concórdia, SC. E-mail: enmuellervet@yahoo.com.br

${ }^{3}$ Médica Veterinária, Clínica Veterinária Novo Esteio, Esteio, RS. E-mail: elisangelafischer@ig.com.br

${ }^{4}$ Prof. Dr. do Centro de Ciências Químicas, Farmacêuticas e de Alimentos, UFPel, Pelotas, RS. E-mail: noty62@hotmail.com

${ }^{5}$ Prof $^{\mathrm{a}} \mathrm{Dr}^{\mathrm{a}}$ do Dept ${ }^{\mathrm{o}}$ de Clínicas Veterinária, UFPel, Pelotas, RS. E-mail: marciaonobre@gmail.com

* Autor para correspondência
} 
$\mathrm{dL}( \pm 10,73)$, while the ones obtained from laboratorial enzymatic method were $80,7 \mathrm{mg} / \mathrm{dL}( \pm 9,97)$, with no statistical differences. It is concluded that the glucometer can be securely used in dogs, since the average values of capillary and venous glycemia, measured with portable digital glucometer, were similar to the values obtained by laboratorial method.

Key words: Glucose, glucometer, spectrophotometry

\section{Introdução}

A glicose é a principal fonte de energia para os animais e humanos. Situações que cursam com diminuição ou aumento nos níveis glicêmicos podem provocar sérias complicações à saúde dos animais (FARIA, 2007). O monitoramento clínico em cães é importante para detecção precoce de alterações da glicemia e no auxílio diagnóstico de doenças decorrentes destas alterações (CRUZ FILHO et al., 2002; FARIA; ARAÚJO; SOTOBLANCO, 2005).

Avaliações periódicas da glicemia podem ser realizadas com auxílio de aparelhos portáteis e por método laboratorial (VANDRESEN et al., 2009; ALEIXO et al., 2010; ARGOLLO; FAUSTINO; PEDREIRA, 2010). Os aparelhos portáteis aferem índices glicêmicos do sangue periférico capilar e venoso (ALEIXO et al., 2007; ARGOLLO; FAUSTINO; PEDREIRA, 2010). Os glicosímetros digitais oferecem uma série de benefícios em relação aos analisadores automáticos-padrões, utilizados em laboratórios de diagnóstico (COHN et al., 2000) entre eles o fato de serem pequenos, portáteis, fáceis de manusear, além de requererem uma pequena quantidade de sangue para se realizar o exame (FOSTER; GOODE; SMALL, 1999; COHN et al., 2000; WESS; REUSCH, 2000a, 2000b). Além disso, o glicosímetro digital portátil é uma opção aplicável para aferição da glicemia em pacientes com amostras lipêmicas, hemolisadas e ictéricas (ALEIXO et al., 2010), as quais podem resultar em erros quando avaliadas pelo método laboratorial.

Estudos avaliando a glicemia de cães hígidos foram realizados por aferição com glicosímetro digital portátil e método enzimático laboratorial (ALEIXO et al., 2010) e ainda, comparando as amostras de sangue periférico capilar e sangue venoso (BOYD; LEIGH; STUART, 2005). Assim sendo, torna-se necessário avaliar e comparar a variação nos níveis séricos determinado por cada técnica ou fonte sanguínea. Desta forma, objetivouse medir e comparar a glicemia de cães hígidos com glicosímetro digital portátil avaliando sangue periférico capilar e venoso, tendo como padrão de referência os valores obtidos pelo método enzimático laboratorial com amostras de sangue venoso.

\section{Material e Métodos}

O estudo foi desenvolvido em 20 cães adultos, com idade entre dois e nove anos, hígidos, pesando entre 5 e $22,5 \mathrm{~kg}$, sem raça definida, sendo 10 fêmeas e 10 machos. Os animais estavam vermifugados e eram alimentados com ração canina industrializada. Todos os animais foram submetidos a jejum alimentar de 12 horas prévias as coletas das amostras sanguíneas. Foi coletado sangue periférico capilar e sangue venoso, para aferição dos níveis de glicose.

A face interna da orelha esquerda de cada animal foi puncionada, com agulha $25 \times 7 \mathrm{~mm}$ (Embramac $\left.{ }^{\circledR}\right)$, para coleta de $6 \mu \mathrm{L}$ de sangue periférico para dosagem glicêmica imediata em glicosímetro digital portátil (Accu-Chek ${ }^{\circledR}$ Performa Nano, utilizando tiras teste Accu-Chek ${ }^{\circledR}$ Performa, ambos da Roche Diagnóstica do Brasil Ltda.), realizado através de reações eletroquímicas, sempre pelo mesmo avaliador. Para a aferição dos níveis de glicose plasmática pelo método enzimático da glicose oxidase (GOD), foram coletados dois $\mathrm{mL}$ de sangue venoso, utilizando seringa descartável de $3 \mathrm{~mL}$ (Injex®) com agulha $25 \times 0,7 \mathrm{~mm}$, através de punção na veia cefálica. As amostras foram 
acondicionadas em tubos contendo anticoagulante EDTA (ácido etilenodiaminotetracético) e fluoreto de sódio e, imediatamente, enviadas para a análise laboratorial, sendo este método considerado padrão-ouro para aferição da glicemia plasmática. Utilizando o sangue venoso residual contido na seringa, avaliou-se a glicemia com glicosímetro digital portátil, conforme as instruções do fabricante quanto ao momento de inserção da tira e o tempo máximo para leitura.

As variáveis foram expressas em média e desvio padrão, sendo os valores de glicose do sangue periférico capilar comparado com os do sangue venoso, ambos obtidos por glicosímetro digital portátil. Os valores glicêmicos obtidos por glicosímetro digital portátil de amostras de sangue periférico capilar também foram comparados aos valores glicêmicos resultantes do emprego do método enzimático laboratorial. Foi utilizada estatística descritiva para definição do coeficiente de variação média entre as duas técnicas de até $20 \%$, aceito pelo International Organization for Standardization. Verificando a existência de normalidade, através do teste de Shapiro-Wilk, utilizou-se coeficiente de correlação de Pearson e ANOVA - teste de Tukey, com intervalo de confiança de 95\%, extraído do programa Statistix 9.0 em todos os casos.

\section{Resultados e Discussão}

Os valores médios da glicemia de cães, aferidos com glicosímetro digital portátil de sangue periférico capilar, venoso e pelo método enzimático laboratorial foram, respectivamente, $84,60 \mathrm{mg} / \mathrm{dL}$ $( \pm 11,15), 76,65 \mathrm{mg} / \mathrm{dL}( \pm 10,73)$ e $80,7 \mathrm{mg} / \mathrm{dL}( \pm$ 9,97) (Figura 1). Estudos em cães normoglicêmicos, utilizando glicosímetro digital portátil, demonstram que os valores séricos de glicose em sangue periférico capilar variam de $76,06 \mathrm{mg} / \mathrm{dL}$ a $77,75 \mathrm{mg} / \mathrm{dL}$ e no sangue venoso de $74,58 \mathrm{mg} / \mathrm{dL}$ a $82,53 \mathrm{mg} / \mathrm{dL}$ (ALEIXO et al., 2007, 2010). Foi observado que os valores obtidos com glicosímetro digital portátil não diferiram estatisticamente entre o sangue periférico capilar e o sangue venoso $(\mathrm{p}=0,78)$, assim como entre os valores obtidos pelo método laboratorial enzimático e glicosímetro digital portátil, a partir do sangue periférico capilar $(p=0,46)$ e venoso $(p=0,64)$. O coeficiente de correlação entre o sangue periférico capilar e o sangue venoso avaliada pelo glicosímetro digital portátil foi considerada fraca $(\mathrm{r}=0,41)$. Em humanos, os valores glicêmicos do sangue periférico capilar e venoso, mensurados com glicosímetro digital portátil e pelo método laboratorial, foram 137,88 mg/dL, 143,82 mg/dL e $127,35 \mathrm{mg} / \mathrm{dL}$, respectivamente, embora tenha havido diferença estatística entre os valores da glicemia capilar e laboratorial $(r=0,97 ; p<0,001)$ e entre venosa e laboratorial $(r=0,96 ; p<0,001)$, a correlação foi considerada forte e a diferença existente foi de $18 \mathrm{mg} / \mathrm{dL}$, a qual pode ter acontecido pela precisão inerente aos aparelhos portáteis desenvolvidos para aferição da glicemia (BOYD; LEIGH; STUART, 2005). Cordova et al. (2009) obtiveram como valores da amostra capilar e venosa aferida por glicosímetro digital portátil 101,52 $\mathrm{mg} / \mathrm{dL}$ e 104,94 mg/dL, respectivamente; e 90,36 $\mathrm{mg} / \mathrm{dL}$ quando a amostra venosa foi aferida por método laboratorial, havendo boa correlação entre a determinação da glicemia utilizando o glicosímetro digital portátil e o método enzimático laboratorial $(r=0,8742 ; p>0,005)$.

Para que não houvesse variação dos níveis de glicose devido ao jejum ou pós-prandial os animais foram mantidos em jejum de 12 horas. Durante o jejum, a glicose capilar pode estar ligeiramente maior $(2-5 \mathrm{mg} / \mathrm{dL})$ que a glicose venosa, sendo os níveis de glicose no sangue arterial apenas 5 $\mathrm{mg} / \mathrm{dL}$ maiores que no sangue capilar e $10 \mathrm{mg} / \mathrm{dL}$ maior que a concentração de glicose no sangue venoso. Isto ocorre porque o sangue arterial, responsável por entregar para os tecidos a glicose que é absorvida e utilizada como fonte de energia, possui maiores níveis de glicose quando comparado com o sangue venoso (BLAKE; NATHAN, 2004). Quando o organismo entra em privação alimentar, a glicose fica retida nos capilares sanguíneos e é 
encontrada em maior quantidade que no restante do organismo. No estágio pós-prandial, entretanto, o sangue capilar pode ser $20-25 \%$ ou mais elevado que o nível de glicose no sangue venoso (FARRER et al., 1995). Outro fator que pode interferir nos resultados é o incorreto manuseio do glicosímetro digital portátil, assim sendo, foram devidamente seguidas as instruções do fabricante do glicosímetro digital portátil e fitas-teste, buscando minimizar os erros e aumentar a acurácia dos resultados, visto que Aleixo et al. (2006) já relataram descarte de amostras a interferência destes fatores.

Figura 1. Valores glicêmicos mínimo, média e máximo em cães após 12 horas de jejum aferidos por glicosímetro digital portátil , reação eletroquímica, utilizando amostra capilar e venosa e por método enzimático laboratorial.

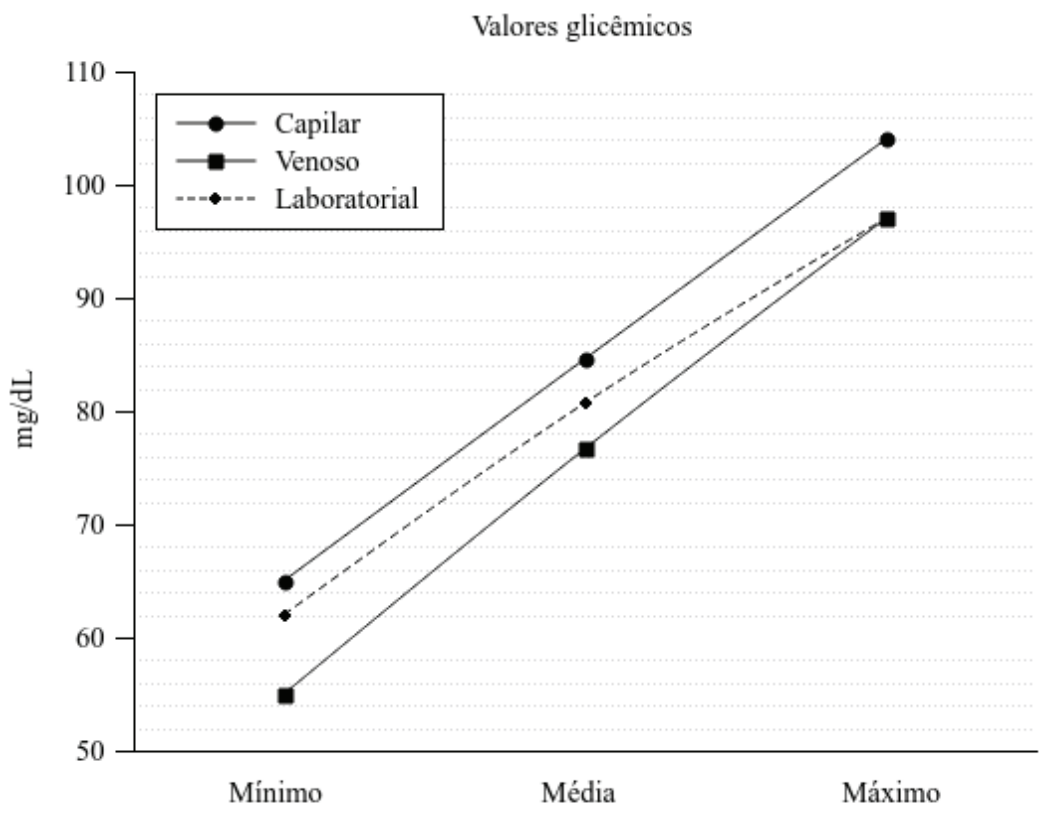

Fonte: Elaboração dos autores.

A variação da média da glicemia obtida pelo glicosímetro digital portátil, na amostra capilar e venosa, foi de $16,6 \%$; na amostra periférica capilar versus método laboratorial foi de $12,6 \%$ e a variação da amostra venosa versus método laboratorial foi de $15 \%$. Vandresen et al. (2009) compararam os valores glicêmicos, obtidos por glicosímetro digital portátil (sistema capilar) e por método laboratorial, de 36 pacientes humanos sendo estes tomados em quatro momentos, e observaram que não houve diferença estatística significativa entre os métodos, embora o teste de Kappa mostrasse maior concordância em diferentes momentos. Da mesma forma, um estudo realizado com 39 gatos hiperglicêmicos, cuja glicemia era superior a162 $\mathrm{mg} / \mathrm{dL}$, mostrou maiores coeficientes de variação na mensuração com glicosímetro $(3,5 \%)$ em relação aos obtidos pelo método laboratorial (0,9\%) (ZINI et al., 2009). Esta variação pode ocorrer porque existem diferenças físicas entre a concentração de glicose no soro/plasma e no sangue total bem como o sangue venoso comparado com o sangue capilar. A glicose se equilibra na porção aquosa da amostra sanguínea, sendo que a concentração de água no soro/plasma difere da concentração de água na porção celular do sangue (BURTIS; ASHWOOD, 1999). Os eritrócitos contêm membranas lipídicas e altos níveis de proteína hemoglobina em seu 
interior que excluem água, portanto a água irá variar de acordo com o hematócrito. Soro/plasma contém mais água e, portanto, alta concentração de glicose sendo aproximadamente 11-12\% comparada com o sangue total (BURTIS; ASHWOOD, 1999). Já foram relatadas variações de 9,4\% entre os valores da glicemia capilar obtida em aparelho portátil e $10,4 \%$ da venosa através do método enzimático laboratorial (ALEIXO et al., 2010).

De acordo com International Organization for Standardization (ISO 15197:2003), os resultados obtidos da glicemia capilar e através de métodos laboratoriais podem sofrer variações de $20 \%$ e isso ocorre porque as medições de glicose analisam todo o sangue (GARG et al., 2004) e estabelecer esta precisão torna-se difícil, pois a glicose é instável em todo sangue. A diferença entre os valores do sangue venoso aferido pelo glicosímetro e pelo método laboratorial pode ocorrer devido a análise imediata obtida com glicosímetro e o tempo inerente gasto com o método laboratorial, pois os eritrócitos metabolizam glicose, o que pode acarretar no decréscimo da concentração da glicose na amostra em 5-7\% por hora, tanto quanto o soro/ plasma se mantiverem em contato com as células vermelhas do sangue (CHAN; SWAMINATHAN; COCKRAM, 1989).

\section{Conclusões}

Conclui-se que o glicosímetro pode ser utilizado com segurança em cães para avaliar a glicemia capilar e venosa, com resultados similares ao laboratorial.

\section{Agradecimentos}

Ao CNPq e a CAPES pelo auxílio financeiro e ao Laboratório Dr. Rouget Perez pelas análises laboratoriais.

\section{Referências}

ALEIXO, G. A. S.; COELHO, M. C. O. C.; GUIMARÃES, A. L. N.; ANDRADE, M. B.; LOPES JÚNIOR, C. G.; SILVA, R. A. A.; CAVALCANTI, H. B. Fatores que podem invalidar os resultados da mensuração dos níveis glicêmicos em cães utilizando o glicosímetro portátil. Ciência Animal Brasileira, Goiânia, v. 7, n. 4, p. 447-454, 2006.

ALEIXO, G. A.; COELHO, M. C.; TENÓRIO, A. P.; SILVA, J. A. A.; GUIMARÃES, A. L. N.; ANDRADE, M. B.; LOPES JUNIOR, C. G.; NASCIMENTO, C. R. A. Mensuração da glicemia em cães mediante a utilização do glicosímetro portátil: comparação entre amostras de sangue capilar e venoso. Medicina Veterinária, Recife, v. 1, n. 1, p. 9-13, 2007.

ALEIXO, G. S.; COELHO, M. C. O.; TENÓRIO, A. P. M.; GUIMARÃES, A. L. N.; ANDRADE, M. B.; CAVALCANTI, H.B. Uso do glicosímetro portátil para determinar a concentração de glicose no sangue de cães. Ciência Animal Brasileira, Goiânia, v. 11, n. 3, p. 537$545,2010$.

ARGOLLO, A. P. B.; FAUSTINO, T. N.; PEDREIRA, L. C. Valores glicêmicos oferecidos pelo glicosímetro portátil, utilizando sangue de diferentes vias de coleta: estudo de validade. Revista Brasileira de Terapia Intensiva, São Paulo, v. 22, n. 4, p. 351-357, 2010.

BLAKE, D. R.; NATHAN, D. M. Point-of-care testing for diabetes. Critical Care Nursing Quarterly, Philadelphia, v. 27, n. 2, p. 150-61, 2004.

BOYD, R.; LEIGH, B.; STUART, P. Capillary versus venous bedside blood glucose estimations. Emergency Medical Journal, British, v. 22, n. 3, p. 177-179, 2005.

BURTIS, C. A.; ASHWOOD, E. R. Tietz textbook of clinical chemistry. $3^{\text {rd }}$ ed. Philadelphia: W.B. Saunders, 1999.

CHAN, A. Y.; SWAMINATHAN, R.; COCKRAM, C. $\mathrm{S}$. Effectiveness of sodium fluoride as a preservative of glucose in blood. Clinical Chemistry, Washington, v. 2, n. 35, p. 315-317, 1989.

COHN, L. A.; MCCAW, D. L.; TATE, D. J.; JOHNSON, J. C. Assessment of five portable blood glucose meters, a point-of-care analyzer, and color test strips for measuring blood glucose concentration in dogs. Journal of the American Veterinary Medical Association, Schaumburg, v. 216, n. 2, p. 198-202, 2000. 
CORDOVA, C. M.; VALLE, J. P.; YAMANAKA, C. N.; CORDOVA, M. M. Determinação das glicemias capilar e venosa com glicosímetro versus dosagem laboratorial da glicose plasmática. Jornal Brasileiro de Patologia e Medicina Laboratorial, Rio de Janeiro, v. 45, n. 5, p. 379-384, 2009.

CRUZ FILHO, R. A.; CORREAA, L. L.; EHRHARDT, A. O.; CARDOSO, G. P.; BARBOSA, G. M. O Papel da glicemia capilar de jejum no diagnóstico precoce do diabetes mellitus: correlação com fatores de risco cardiovascular. Arquivo Brasileiro de Endocrinologia e Metabolismo, São Paulo, v. 46, n. 3, p. 255-259, 2002.

FARIA, P. F. Diabetes mellitus em cães. Acta Veterinária Brasílica, Mossoró, v. 1, n. 1, p. 8-22, 2007.

FARIA, P. F.; ARAÚJO, D. F.; SOTO-BLANCO, B. Glicemia em cães obesos e senis. Acta Scientiae Veterinariae, Porto Alegre, v. 33, n. 1, p. 47-50, 2005.

FARRER, M.; ALBERS, C. J.; NEIL, H. A.; ADAMS, P. C.; LAKER, M. F.; ALBERTI, K. G. Assessing the impact of blood sample type on the estimated prevalence of impaired glucose tolerance and diabetes mellitus in epidemiological surveys. Diabetic Medicine, Hoboken, v. 12 , n. 4, p. 325-329, 1995.

FOSTER, S. A.; GOODE, J. K. R.; SMALL, R. E. Home blood glucose monitoring. The Annals of Pharmacotherapy, Cincinnati, v. 33, n. 3, p. 355-363, 1999.
GARG, S. K.; CARTER, J. A.; MULLEN, L.; FOLKER, A. C.; PARKES, J. L.; TIDEMAN, A. M. The Clinical performance and ease of use of a blood glucose meter that uses a 10-test disk. Diabetes Technology \& Therapeutics, Nova Iorque, v. 6, n. 4, p. 495-502, 2004.

VANDRESEN, L. T. S.; SCHNEIDER, D. S. L. G.; BATISTA, M. R.; CROZATTI, M. T. L.; TEIXEIRA, J. J. V. Níveis glicêmicos de pacientes diabéticos segundo estudo comparativo entre duas técnicas. Revista de Ciências Farmacêuticas Básica e Aplicada, Araraquara, v. 30, n. 1, p. 111-113, 2009.

WESS, G.; REUSCH, C. Capillary blood sampling from the ear of dogs and cats and use os portable meters to measure glucose concentration. Journal of Small Animal Practice, Malden, v. 41, n. 2, p. 60-66, 2000 b.

Evaluation of five portable blood glucose meters for use in dogs. Journal of the American Veterinary Medical Association, Schaumburg, v. 216, n. 2, p. 203209, jan. 2000a.

ZINI, E.; MORETTI, S.; TSCHUOR, F.; REUSCH, C. E. Evaluation of a new portable glucose meter designed for the use in cats. Clinic for small animal internal medicine, German, v. 151, n. 9, p. 448-51, 2009. 